\title{
CORRIGENDUM
}

\section{Optical Image Generation and High-precision Line-of-Sight Extraction for Mars Approach Navigation - CORRIGENDUM}

\author{
Xiuqiang Jiang, Shuang Li, Long Gu, Jun Sun, and Dongdong Xiao
}

doi:10.1017/S0373463318000450, Published by Cambridge University Press, 3 July 2018.

First published online: 30 October 2018 .

The authors of "Optical Image Generation and High-precision Line-of-Sight Extraction for Mars Approach Navigation" first published online on 3 July 2018 (Jiang et al., 2018) would like to make the following corrections to the paper. In particular, the authors apologise to Dr John A Christian for not citing his work (Christian, 2017) in the appropriate places in the paper.

1. On page 1, the sentence in the Abstract, "Next, a hybrid image processing and subpixellevel line-of-sight extraction algorithm is proposed through modification of moment-based sub-pixel edge detection and improvement of direct least-square fitting approaches." should be revised as "Next, a hybrid image processing and line-of-sight extraction algorithm is proposed through objective segmentation and rough edge detection, pseudo-edge elimination and precise edge detection, and robust ellipse fitting."

2. In the last paragraph on page 10, the citation "(Du et al., 2016; Christian, 2017)" should be revised as "(Ghosal and Mehrotra, 1993; Du et al., 2016)"; a citation "(Christian, 2017)" should be added to the end of the sentence "Therefore, a ramp model is introduced to modify the traditional Zernike moment-based sub-pixel edge detection algorithm" and the end of this paragraph. The reference "Ghosal, S. and Mehrotra, R. (1993). Orthogonal Moment Operators for Subpixel Edge Detection. Pattern Recognition, 26(2), 295-306." should be added to the reference list.

3. On page 11, a citation "(Christian, 2017)" should be added to the end of the caption "Figure 7. Sketch of two-dimensional sub-pixel edge models"; a sentence "As the subsequent operations in this step, a modified Zernike moments technique based on the intensity ramp edge model (Christian, 2017) is employed to conduct sub-pixel edge detection, which is reviewed as follows." should be added to the beginning of the first paragraph.

4. On page 22, the last sentence of the second paragraph in Section 4.4, "The proposal benefits from the modification of moment-based sub-pixel edge detection and improvement of direct least-square fitting approaches." should be revised as "The proposal benefits from the effective collaboration of rough edge detection, precise edge detection, and robust ellipse fitting." 
5. On page 23, the sentence in the Section 5 Conclusions, "A new line-of-sight extraction technique is proposed through the modification of moment-based sub-pixel edge detection and improvement of direct least-square fitting approaches." should be revised as "A hybrid line-of-sight extraction technique is developed through successively conducting objective segmentation and rough edge detection, pseudo-edge elimination and precise edge detection, and robust ellipse fitting."

\section{REFERENCES}

Christian, J.A. (2017). Accurate Planetary Limb Localization for Image-Based Spacecraft Navigation. Journal of Spacecraft and Rockets, 54(3), 708-729.

Du, S., Wang, M., Chen, X., Fang, S. and Su, H. (2016). A High-accuracy Extraction Algorithm of Planet Centroid Image in Deep-Space Autonomous Optical Navigation. The Journal of Navigation, 69(4), 828-844.

Ghosal, S., and Mehrotra, R., (1993). Orthogonal Moment Operators for Subpixel Edge Detection. Pattern Recognition, 26(2), 295-306.

Jiang, X., Li, Shuang, Gu, L., Sun, J. and Xiao, D. (2018). Optical Image Generation and High-precision Line-ofSight Extraction for Mars Approach Navigation. The Journal of Navigation, doi:10.1017/S0373463318000450. 